\title{
Diabetes, glaucoma, sex, and cataract: analysis of combined data from two case control studies
}

\author{
John J Harding, Muriel Egerton, Ruth van Heyningen, Ruth S Harding
}

\begin{abstract}
Data from two case control studies in Oxfordshire were combined and analysed. The combined study covered 1940 subjects, 723 cases, and 1217 controls, between the ages of 50 and 79 with a response rate of $97 \%$ for cases and $94 \%$ for controls. Diabetes was shown to be a powerful and highly significant risk factor for cataract with a relative risk of 5.04. More than $11 \%$ of cataracts in Oxfordshire are attributable to diabetes. The relative risk did not increase significantly with age within the range $\mathbf{5 0}$ to 79 years but was higher in females than in males. For females with diabetes the relative risk was 7.85 with $95 \%$ confidence interval from 4.30 to 14.3 compared with 3.42 with confidence interval from 2.05 to 5.71 for males with diabetes. Diabetes remained a powerful risk factor when other identified risk factors had been controlled for. No known mechanism for the development of diabetic complications provides an explanation for the excess risk in females. Combination of the two studies led to better estimates of the relative risk of glaucoma as a risk factor for cataract (3.96 with $95 \%$ confidence interval from 2.35 to 6.68). The relative risk appeared to be greater in women than in men but this difference was not statistically significant. There was no significant change in risk with age. Glaucoma is a powerful and independent risk factor for cataract in both sexes and may be responsible for $5 \%$ of all cataracts in our area.

(Brf Ophthalmol 1993; 77: 2-6)
\end{abstract}

Cataract is an important complication of diabetes, and diabetes is the most important risk factor for cataract identified in Western countries. Diabetes has been reported to increase the risk of cataract up to 12-fold in different populations. ${ }^{1-9}$ Further evidence that diabetes can cause cataract is derived from observations of cataract development in experimental diabetes and from in vitro studies of the effects of high sugar concentrations on incubated lenses and lens proteins. ${ }^{10}$

In some of the epidemiological studies the subjects were subdivided by sex for analysis and this indicated an enhanced risk of cataract in females with diabetes than in males with diabetes in the HANES study from the United States, ${ }^{2}$ in
Denmark, ${ }^{3}$ and in England..$^{6-8}$ The confidence intervals for each sex, where given, however, were wide and the difference between the sexes was not statistically significant. For example in our own studies the relative risks (and confidence intervals) for diabetic males and females were $3 \cdot 2$ $(1.3$ to 7.6$)$ and $10.5(4.5$ to 24.6$)$ respectively in the first study, ${ }^{6}$ and $3.4(1.8$ to 6.4$)$ and $6.0(2.6$ to $14 \cdot 2)$ in the second study. ${ }^{8}$

Individually each of these results identified diabetes as a highly significant and powerful risk factor in males and females separately, but the difference between the sexes was not significant, although there were 300 cataract patients in the first study and 423 in the second.

The possibility that glaucoma or treatment of glaucoma leads to cataract has been investigated for many years with the more recent emphasis on glaucoma surgery as a cause of cataract. ${ }^{3811-16}$ It has been estimated that glaucoma increases the risk of cataract by up to sixfold in different populations over the age of $50 .^{3814}$ No difference between the sexes was reported. ${ }^{314}$

A claimed decrease in relative risk with age ${ }^{3}$ was not entirely convincing above the age of 50 and very few glaucoma patients were identified below that age.

Glaucoma was identified as a risk factor for cataract in the two case control studies in Oxfordshire with relative risks of 5.95 with a $95 \%$ confidence interval from 2.6 to 13.5 in the first, ${ }^{14}$ and relative risk of 2.9 with a $95 \%$ confidence interval from 1.5 to 5.7 in the second. ${ }^{8}$ The relative risks seem different but the confidence intervals overlap.

These two studies covered almost 1000 patients each. Both were based on interviews of all the patients and a larger group of age and sexmatched controls. Some questions were the same in both studies and so it has been possible to pool the data on diabetes, glaucoma, and on several other major risk factors from the two studies, giving information on a total of 1940 subjects. The large numbers should permit a more accurate assessment of the relative risk for diabetes and glaucoma, an exploration of differences in risk between the sexes and between age groups, and the assessment of attributable risk. This paper reports the analysis of data from the combined studies in relation to the association between diabetes, glaucoma, and cataract. It has been possible to establish clearly for the first time that the risk of cataract associated with diabetes is greater in females than in males. 
Table 1 Number (\%) of male and female cases and controls in each age group

\begin{tabular}{llllrrr}
\hline & \multicolumn{6}{c}{ Age groups } \\
\cline { 2 - 7 } & $50-54$ & $55-59$ & $60-64$ & $65-69$ & \multicolumn{1}{c}{$70-74$} & \multicolumn{1}{c}{$75-79$} \\
\hline Males: & & & & & & \\
Controls & $23(4 \cdot 2)$ & $54(9 \cdot 8)$ & $81(14 \cdot 7)$ & $95(17 \cdot 2)$ & $151(27 \cdot 4)$ & $148(26 \cdot 8)$ \\
Cases & $13(3 \cdot 8)$ & $33(9 \cdot 6)$ & $50(14 \cdot 6)$ & $59(17 \cdot 3)$ & $94(27 \cdot 5)$ & $93(27 \cdot 2)$ \\
Females: & $36(5 \cdot 4)$ & $49(7 \cdot 4)$ & $97(14 \cdot 6)$ & $115(17 \cdot 3)$ & $172(25 \cdot 9)$ & $196(29 \cdot 5)$ \\
Controls & $16(4 \cdot 2)$ & $26(6 \cdot 8)$ & $50(13 \cdot 1)$ & $63(16 \cdot 5)$ & $103(27 \cdot 0)$ & $123(32 \cdot 3)$ \\
Cases & 16 & & & & & \\
\hline
\end{tabular}

\section{Subjects and methods}

The subjects, 723 cases and 1217 controls, were pooled from the two original case control studies of cataract in Oxfordshire. ${ }^{814}$ Cases were those aged 50 to 79 years admitted to the Oxford Eye Hospital for cataract extraction. Controls were recruited both from other hospital departments and from the age-sex registers of general practitioners. As the Oxford Eye Hospital is a National Health Service hospital with a catchment population of about 560000 and the vast majority of the population are registered with general practitioners, the cases and controls in the two studies were thought to be representative of all cataract patients, and of the general population respectively. The controls were chosen to give an agesex distribution that matched the cases. The overall response rates were $97 \%$ for cases and $94 \%$ for controls with the non-responders including those too ill to be interviewed and those who spoke no English.

The cases and controls were interviewed in the same way by the same interviewers, using the same forms. ${ }^{814}$ The questionnaires included questions on medical history and ocular history, and on drug therapy. In the second study selfreported glaucoma was confirmed by examination of the hospital notes after completion of interviewing. ${ }^{8}$ This supported the reliability of the self-reporting technique.

In relation to this paper information on diabetes was ascertained when patients were asked if

Table 2 Diabetes as a risk factor for cataract

\begin{tabular}{lclr}
\hline & Controls & Cases & Total \\
\hline No diabetes & 1179 & 622 & 1801 \\
Diabetes & 38 & 101 & 139 \\
Total & 1217 & 723 & 1940 \\
Percent positive & $3 \cdot 1$ & 13.9 &
\end{tabular}

$\chi^{2}=80 \cdot 2 ; \mathrm{p}<<0.001$.

Relative risk $=5 \cdot 04 ; 95 \%$ confidence interval 3.43 to $7 \cdot 41$.

Table 3 Diabetes as a risk factor in males

\begin{tabular}{lccc}
\hline & Controls & Cases & Total \\
\hline No diabetes & 528 & 296 & 824 \\
Diabetes & 24 & 46 & 70 \\
Total & 552 & 342 & 894 \\
Percent positive & 4.4 & 13.4 & \\
\hline
\end{tabular}

$\chi^{2}=24 \cdot 2 ; \mathrm{p}<<0.001$.

Relative risk $=3 \cdot 42 ; 95 \%$ confidence interval 2.05 to $5 \cdot 71$.

Table 4 Diabetes as a risk factor in females

\begin{tabular}{lccr}
\hline & Controls & Cases & Total \\
\hline No diabetes & 651 & 326 & 977 \\
Diabetes & 14 & 55 & 69 \\
Total & 665 & 381 & 1046 \\
Percent positive & $2 \cdot 1$ & 14.4 & \\
\hline
\end{tabular}

$\chi^{2}=59.8 ; \mathrm{p}<<0.001$.

Relative risk $=7 \cdot 85 ; 95 \%$ confidence interval $4 \cdot 30$ to $14 \cdot 3$. they had ever suffered a serious illness and if they had been admitted to hospital. Positive responses were followed up and diabetes was coded on the questionnaire. In the second study those reporting diabetes were asked its duration. Confirmation of diabetes was not sought. Blood analysis would have been difficult when subjects were interviewed at home up to 30 miles $(50 \mathrm{~km})$ from Oxford and would have decreased our response rate. Confirmation via clinical notes would have been invalid as different sources, hospital and general practitioners, would have had to be used for different subjects leading to ascertainment bias. The similarity in the results between the two studies and between the different control groups in each study indicates that we were getting unbiased data for known diabetes.

Data were entered into the university's Vax computer. In the present study data files from the two original studies were combined before analysis initially by the $\chi^{2}$ test for categorical variables using the SAS statistical package (SAS Institute Inc, Cary, NC, USA). Odds ratios were calculated from contingency tables and taken as valid estimates of relative risk. Confidence intervals and attributable risks were calculated as described. ${ }^{8}$ Interactions between risk factors were explored using generalised linear modelling (GLIM: Royal Statistical Society, London).

\section{Results}

The control subjects were partly hospital controls and partly community controls. Each control group, four groups in the first study and three in the second, was matched to the cases for age and sex distribution so the overall match is very close (Table 1). Results for the different control groups were similar in the two original studies and so all were pooled for this combined analysis.

Diabetes appeared as a powerful risk factor in

Table 5 Diabetes as a risk factor for cataract in subjects aged 50 to 69 years

\begin{tabular}{lccc}
\hline & Controls & Cases & Total \\
\hline No diabetes & 537 & 267 & 804 \\
Diabetes & 13 & 43 & 56 \\
Total & 550 & 310 & 860 \\
Percent positive & $2 \cdot 4$ & 13.9 & \\
\hline
\end{tabular}

$\chi^{2}=43 \cdot 1 ; p<<0.001$.

Relative risk $=6.65 ; 95 \%$ confidence interval 3.52 to 12.6 .

Table 6 Diabetes as a risk factor in subjects aged 70 to 79 years

\begin{tabular}{lccr}
\hline & Controls & Cases & Total \\
\hline No diabetes & 642 & 355 & 994 \\
Diabetes & 25 & 58 & 83 \\
Total & 667 & 413 & 1080 \\
Percent positive & 3.8 & 14.0 & \\
\hline
\end{tabular}

$\chi^{2}=38 \cdot 1 ; p<<0.001$

Relative risk $=4 \cdot 20 ; 95 \%$ confidence interval $2 \cdot 58$ to $6 \cdot 83$.

Table 7 Diabetes as a risk factor for cataract in three age groups

\begin{tabular}{llll}
\hline Age & Relative risk & Confidence interval & $p$ \\
\hline $50-59$ & 12.6 & $2 \cdot 76-57.9$ & $<0.001$ \\
$60-69$ & 5.56 & $2 \cdot 74-11.3$ & $<0.001$ \\
$70-79$ & $4 \cdot 20$ & $2.58-6.83$ & $<0.001$
\end{tabular}

Breslow-Day homogeneity test: $\chi^{2}=2 \cdot 1 ; p=0 \cdot 35$. 
the combined study with $\chi^{2}$ higher than before and the confidence interval somewhat narrower (Table 2). The relative risk of 5.04 compares with $6 \cdot 2$ found in the first Oxford study ${ }^{6}$ and $4 \cdot 2$ found in the second. ${ }^{8}$ In the separate studies the relative risks for female diabetics were two to three times those for male diabetics. Analysing the combined data diabetes is a powerful risk factor for cataract in both males (Table 3) and females (Table 4) separately. Both confidence intervals are well above unity showing a clear risk association. The difference between the sexes was tested by the Breslow-Day test for homogeneity $^{17}$ which gave $\chi^{2}=4.31$ and $\mathrm{p}=0.038$ showing that the relative risks are significantly different for males and females with diabetes. The interaction between sex and diabetes determined in a GLIM analysis was also statistically significant $\left(\chi^{2}=4 \cdot 1 ; \mathrm{p}<0.05\right)$. The excess risk for females with diabetes over that for males with diabetes was $2 \cdot 294$ ( $95 \%$ confidence inter$\mathrm{val}=1 \cdot 023$ to $5 \cdot 143$ ). In other words diabetes in females is a more powerful risk factor for cataract than diabetes in males. Nevertheless diabetes is a risk factor in both sexes.

Some earlier studies have determined the relative risk or odds ratio for diabetes as a risk factor for cataract at different ages and claimed a decline with age though without showing the agerelated decline in risk was statistically significant. ${ }^{23}$ The numbers of cataract patients and controls in this combined analysis have enabled us to look again at the age relationship. First we divided the subjects into two groups of similarsize: ages 50 to 69 and ages 70 to 79 . Diabetes was a highly significant risk factor for cataract in each of these age groups (Tables 5 and 6 ) with a relative risk of 6.7 in the younger group falling to 4.2 in the older group. The Breslow-Day test of homogeneity shows that the difference between the groups is not statistically significant $\left(\chi^{2}=1 \cdot 28 ; \mathrm{p}=0 \cdot 26\right)$. Subdividing by decades gives an impression of an age-related fall in risk but the numbers become small, the confidence intervals wide, and the homogeneity test showed no difference between the three relative risks (Table 7). With 723 cataract patients and 1217 controls there is an apparent age-related decline in risk of cataract associated with diabetes but the decline is not significant. The risk in each age group separately is highly significant.

Although diabetes emerges as a powerful risk factor in the approach described above it was necessary to examine the effect of other risk factors on the risk associated with diabetes using a linear modelling program. The risk factors that remained significant and were incorporated into the final model were diabetes, glaucoma, severe diarrhoea, and myopia. Analysis for glaucoma follows in this paper and that for the other factors will be published separately. The four factors did not interact significantly with each other and the presence of the other three in the model made little difference to the relative risk associated with diabetes - final value $5 \cdot 10$ with a $95 \%$ confidence interval from 3.43 to 7.58 (compare Table 2). The self-reporting of glaucoma was checked and confirmed by examination of hospital notes. ${ }^{8}$ Glaucoma emerged as a powerful risk factor in the combined study increasing the risk of cataract fourfold (Table 8). As earlier studies had not established a possible excess risk in females with glaucoma, ${ }^{314}$ we analysed the data for the two sexes separately. Glaucoma emerged as a powerful risk factor for cataract in males alone (Table 9) and in females alone (Table 10) with relative risks of 2.6 and 6.6 respectively. The relative risks indicated that females with glaucoma might experience a greater risk of cataract than males with glaucoma, but when the data were analysed for homogeneity between the sexes it just failed to reach statistical significance (Table 10). In a similar way we subdivided the data by age to identify any age-related change in risk. This is not to investigate aging as a risk factor which is not possible in an age-matched study such as this. Glaucoma appeared as a powerful risk factor for cataract in those aged less than 70 (Table 11; relative risk $=2.9$ ) and in the older group (Table 12 ; relative risk $=4 \cdot 5$ ). The Breslow-Day test for homogeneity gave $p=0.45$,

Table 8 Glaucoma as a risk factor for cataract

\begin{tabular}{lccc}
\hline & Controls & Cases & Total \\
\hline Without glaucoma & 1196 & 676 & 1872 \\
With glaucoma & 21 & 47 & 68 \\
Total & 1217 & 723 & 1940 \\
Percent positive & 1.7 & 6.5 & \\
\hline
\end{tabular}

$\chi^{2}=30.6 ; \quad \mathrm{p}<0.001$.

$\chi^{2}=30.6 ; \mathrm{p}<0.001$
Relative risk $=3.96 ; 95 \%$ confidence interval from 2.35 to 6.68 .

Table 9 Glaucoma as a risk factor for cataract in men

\begin{tabular}{lccc}
\hline & Controls & Cases & Total \\
\hline Without glaucoma & 538 & 320 & 858 \\
With glaucoma & 14 & 22 & 36 \\
Total & 552.5 & 342 & 894 \\
Percent positive & 2.5 & 6.4 & \\
\hline
\end{tabular}

$\chi^{2}=8.30 ; p=0.004$

Relative risk $=2 \cdot 64 ; 95 \%$ confidence interval from $1 \cdot 33$ to $5 \cdot 24$.

Table 10 Glaucoma as a risk factor for cataract in women

\begin{tabular}{lccc}
\hline & Controls & Cases & Total \\
\hline Without glaucoma & 658 & 356 & 1014 \\
With glaucoma & 7 & 25 & 32 \\
Total & 665 & 381 & 1046 \\
Percent positive & $1 \cdot 1$ & 6.6 & \\
\hline
\end{tabular}

$\chi^{2}=2.48 ; \mathrm{p}<0.001$.

Relative risk $=6.60 ; 95 \%$ confidence interval from 2.83 to $15 \cdot 4$. Breslow-Day test for homogeneity ${ }^{12}$ (betwen men and women) $\chi^{2}=2 \cdot 77 ; p=0.096$.

Table 11 Glaucoma as a risk factor in the age range 50 to 69 years

\begin{tabular}{lccc}
\hline & Controls & Cases & Total \\
\hline Without glaucoma & 543 & 299 & 842 \\
With glaucoma & 7 & 11 & 18 \\
Total & 550 & 310 & 860 \\
Percent positive & $1 \cdot 3$ & 3.6 & \\
\hline
\end{tabular}

$\chi^{2}=5.01 ; p=0.025$.

Relative risk $=2 \cdot 85 ; 95 \%$ confidence interval from $1 \cdot 10$ to $7 \cdot 44$.

Table 12 Glaucoma as a risk factor in septuagenarians

\begin{tabular}{lccc}
\hline & Controls & Cases & Total \\
\hline Without glaucoma & 653 & 377 & 1030 \\
With glaucoma & 14 & 36 & 50 \\
Total & 667 & 413 & 1080 \\
Percent positive & $2 \cdot 1$ & $8 \cdot 7$ & \\
\hline
\end{tabular}

$\chi^{2}=25 \cdot 3 ; \mathrm{p}<0.001$.

Relative risk $=4 \cdot 45 ; 95 \%$ confidence interval from $2 \cdot 37$ to $8 \cdot 36$. Breslow-Day test for homogeneity (between younger and older groups) gave $\chi^{2}=0.58 ; p=0.45$. 
indicating that the apparent increased risk in glaucoma patients with age was not significant.

The possibility that glaucoma appears as a risk factor only because it is associated with other factors was examined in the two original studies. ${ }^{818}$ It has been re-examined using the combined data. Four major risk factors remained in the final model: glaucoma, myopia, diabetes, and diarrhoea. There was no confounding with glaucoma and indeed the relative risk was close to the uncorrected value given in Table $8: 3 \cdot 90$ with a confidence interval from $2 \cdot 26$ to $6 \cdot 71$. Glaucoma is a powerful, independent, and highly significant risk factor for cataract.

It is clear that diabetes and glaucoma are powerful risk factors increasing the risk of cataract fivefold and fourfold in the population as a whole, but it is also of interest to know what proportion of cataract is attributable to these factors. We have examined this by calculations of attributable risk. ${ }^{17}$ This has seldom been calculated. The figure for diabetes from the combined data in Table 2 is $11 \cdot 2 \%$, and values of population attributable risk for males, females, older, and younger subjects are between $10 \cdot 7 \%$ and $12 \cdot 9 \%$ with little difference between them for the groups in Tables 3 to 6 . This indicates that about $11 \%$ of all cataracts would be prevented if diabetes, or at least the cataractogenic effect of diabetes, were eliminated. This makes diabetes the most important risk factor for cataract in Western populations. Finally we calculated the proportion of cataract in Oxfordshire attributable to glaucoma. This worked out to $4.9 \%$. In septuagenarians, in whom glaucoma is more common and the relative risk appears slightly higher, the attributable risk is $6 \cdot 8 \%$.

\section{Discussion}

The choice of controls and possible sources of bias were discussed in the papers on the two separate studies. ${ }^{814}$ Combining those studies has provided data on a greater number of patients with severe cataract, sufficiently impairing to require surgery, than previous studies. This has provided a more accurate figure for the relative risk of cataract associated with diabetes, and has for the first time demonstrated a significant excess risk in female diabetics over male diabetics.

Cataract in subjects with diabetes in the age group studied is not the pure diabetic cataract seen in years gone by but is simply a component of age-related cataract. Diabetes in experimental animals produces cataract and many in vitro experiments bear witness to the damaging effects of glucose and other sugars on the lens. ${ }^{10}$ The combination of epidemiological and laboratory evidence establishes without doubt the causal relationship from diabetes to cataract. How diabetes causes cataract is a more open question. Several routes have been discussed with the most interest having followed the osmotic effect of sorbitol and non-enzymic glycosylation (glycation). The sorbitol mechanism presupposes the accumulation of sorbitol in the lens to levels high enough to exert an osmotic effect and damage lens cells. ${ }^{19}$ Sorbitol is found in cataract lenses from diabetics but the concentrations (median less than $1 \mathrm{mM}$ ) would scarcely balance the excess glucose in the aqueous humour. ${ }^{20}$ No significant accumulation of sorbitol is found in human lens incubated in high glucose media. ${ }^{21-23}$ Glycation of lens proteins is increased in human cataracts from diabetic subjects relative to those from non-diabetics and relative to normal lenses $^{24-29}$ and may therefore appear to be the more plausible mechanism. There are probably additional mechanisms, however, because neither mechanism has been able to explain all the biochemical changes in lenses of diabetic animals notably the early loss of glutathione. ${ }^{10}$ Now we have the additional information that diabetic females have a greater risk of cataract than diabetic males. None of the currently postulated mechanisms for diabetic complications help to explain this discrepancy between the sexes. Other diabetic complications do not appear to affect females more than males; for example the risk of retinopathy is no greater in females than in male diabetics. ${ }^{30}$

One potential problem relevant to this paper is the use of interviews to identify subjects with glaucoma, but in one of the original studies this was confirmed by subsequent examination of the hospital notes. ${ }^{8}$ All subjects reporting glaucoma had indeed been treated for glaucoma in Oxford.

As more studies provide evidence associating glaucoma and cataract it becomes more convincing that glaucoma in some way causes cataract. The present analysis shows a highly significant association indicating a fourfold increase in the risk of visually impairing cataract in those with glaucoma. Glaucoma is a powerful risk factor in both men and women with a possible excess risk in females although that excess is not statistically significant. It remains a powerful risk factor throughout the age range 50 to 79 years.

Drainage surgery frequently leads to cataract $^{311-131516}$ and in Oxford most of the risk associated with glaucoma has been attributed to surgical procedures. ${ }^{8}$ As $5 \%$ of total cataract can be attributed to glaucoma it seems that almost $5 \%$ of cataract surgery would be avoided if the trauma of glaucoma surgery could be avoided.

We thank the TFC Frost Charitable Trust, the Royal National Institute for the Blind and Research into Ageing for financial support. We are grateful to consultants of the Oxford Eye Hospital, Ear, Nose and Throat Department and the Slade Hospital, and to-local general practitioners for access to their patients. Useful discussions with European colleagues were made possible by funds from EURAGE.

1 Caird FI. Problems of cataract epidemiology with special reference to diabetes. Ciba Fdn Symp 1973; 19: 281-301. 2 Ederer F, Hiller R, Taylor HR. Senile lens changes and diabetes in two population studies. Am $\mathcal{F}$ Ophthalmol 1981; 91: 381-95.

3 Bernth-Petersen P, Bach E. Epidemiologic aspects of cataract surgery, III: Frequencies of diabetes and glaucoma in a cataract population. Acta Ophthalmol 1983; 61: 406-16.

4 Schwab IR, Dawson CR, Hoshiwara I, Szuter CF, Knowler WC. Incidence of cataract extraction in Pima Indians. Diabetes as a risk factor. Arch Ophthalmol 1985; 103: 20812.

5 Clayton RM, Cuthbert J, Seth J, Phillips CI, Bartholomew RS, Reid JM. Epidemiological and other studies in the assessment of factors contributing to cataractogenesis. Ciba Fdn ment of factors contrib

6 van Heyningen $\mathbf{R}$, Harding JJ. Risk factors for cataract: diabetes, myopia and sex. Colloq d'INSERM 1986; 147: 381-5.

7 Chen TT, Hockwin O, Dobbs R, Knowles W, Eckerskorn U. Cataract and health status: a case-control study. Ophthalmic Res 1988; 20: 1-9.

8 Harding JJ, Harding RS, Egerton M. Risk factors for cataract in Oxfordshire: diabetes, peripheral neuropathy, myopia, glaucoma and diarrhoea. Acta Ophthalmol 1989; 67: 510-7. 
9 Szmyd L, Schwartz B. Association of systemic hypertension and diabetes mellitus with cataract extraction: a case-control study. Ophthalmology 1989; 96: 1248-52.

10 Harding JJ. Cataract: biochemistry, epidemiology and pharmacology. Chapman and Hall: London, 1991.

11 Shaffer RN, Rosenthal G. Comparison of çataract incidence in normal and glaucomatous populations. Am $\mathcal{F}$ Ophthalmol 1970; 69: 368-70.

12 Laatikainen $L$. Late results of surgery on eyes with primary glaucoma and cataract. Acta Ophthalmol 1971; 49: 281-92.

13 Layden WE. Cataracts and glaucoma. In: Duane TD, Jaeger EA, eds. Clinical ophthalmology, Vol 3. Harper and Row: Philadelphia, 1987: Chap 55: 1-22.

14 van Heyningen R, Harding J J. A case-control study of cataract in Oxfordshire: some risk factors. Brf Ophthalmol 1988; 72: 804-8.

15 Kanski JJ. Clinical ophthalmology, 2nd Ed. London: Butterworth, 1989.

16 Clarke MP, Vernon SA, Sheldrick JH. The development of cataract following trabeculectomy. Eye 1990; 4: 577-83.

17 Breslow NE, Day NE. Statistical methods in cancer research. Vol 1. The analysis of case-control studies. International Agency for Research on Cancer: Lyon, 1980.

18 Harding JJ, van Heyningen R. Risk factors for cataract. $\mathrm{Br} \mathcal{F}$ Ophthalmol 1989; 73: 579-80.

19 Kador PF, Kinoshita JH. Diabetic and galactosaemic cataracts. Ciba Fdn Symp 1984; 106: 110-31.

20 Pirie A, van Heyningen R. The effect of diabetes on the content of sorbitol, glucose, fructose and inositol in the human lens. Exp Eye Res 1964; 3: 124-31.

21 Cousins JP, Aguayo JB, Gottsch JD, Camli U, Hay A, Stark WJ. ${ }^{13} \mathrm{C}$-Nuclear magnetic resonance spectroscopy of glucose metabolism in the human lens. Invest Ophthalmol Vis $\mathrm{Sci}$ 1985; 26 (Suppl): 314.

22 Wolfe JK, Chylack LT. Glucose metabolism by human cataracts in culture. Exp Eye Res 1986; 43: 243-9.

23 Lerman S, Moran M. Sorbitol generation and its inhibition by Sorbinil in the aging normal human and rabbit lens and human diabetic cataracts. Ophthalmic Res 1988; 20: $348-52$

24 Pande A, Garner WH, Spector A. Glucosylation of human lens protein and cataractogenesis. Biochem Biophys Res Commun 1979; 89: 1260-66

25 Ansari NH, Awasthi YG, Srivastava SK. Role of glycosylation in protein disulphide formation and cataractogenesis. $\operatorname{Exp}$ Eye Res 1980; 31: 9-19.

26 Kasai K, Nakamura T, Kase N, Hiraoka T, Suzuki R, Kogure $\mathrm{F}$, et al. Increased glycosylation of proteins from cataractous lenses in diabetes. Diabetologia 1983; 25: 36-8.

27 Rao GN, Cotlier E. Free epsilon amino groups and 5-hydroxymethylfurfural contents in clear and cataractous human methylfurfural contents in clear and cataractous

28 Liang JN, Hershorin LL, Chylack LT. Non-enzymic glycosylation in human diabetic lens crystallins. Diabetologia 1986; 29: $225-8$

29 Oimomi M, Maed Y, Hata F, Kitamura Y, Matsumoto S, Baba $S$, et al. Glycation of cataractous lens in non-diabetic senile subjects and in diabetic patients. Exp Eye Res 1988; 46: 41520.

30 Klein R, Klein BEH, Moss SE, Davis MD, DeMets DL. The Wisconsin epidemiologic study of diabetic retinopathy. II. Prevalence and risk of diabetic retinopathy when age at diagnosis is less than 30 years. Arch Ophthalmol 1984;102. $520-6$. 